\section{THU0602 TARGETING GENERATION Z: DEVELOPMENT AND VALIDATION OF AN ILLUSTRATED TRANSITION READINESS QUESTIONNAIRE FOR ADOLESCENTS WITH JUVENILE IDIOPATHIC ARTHRITIS}

H. Lotfy ${ }^{1}$, M. El Gaafary ${ }^{2}$, N. El Aroussy ${ }^{3}$, D. Mekkawy ${ }^{3}$, Y. Farag ${ }^{1}$, Y. EI Miedany ${ }^{3,4}$, on behalf of PRINTO Egypt. ${ }^{1}$ Pediatrics, School of Medicine Cairo University; ${ }^{2}$ Community and Public Health; ${ }^{3}$ Rheumatology and Rehabilitation, School of Medicine Ain Shams University, Cairo, Egypt, ${ }^{4}$ Rheumatology, Darent Valley Hospital, Dartford, UK

Objectives: To develop, validate and implement a transition readiness assessment tool to be completed by adolescents living with juvenile idiopathic arthritis. Methods: Cross sectional, quantitative multicenter study. Patients with juvenile idiopathic arthritis aged 14 years or older were included. The assessment tool, designed to be self-administered, is divided into 4 domains: medical, psychosocial, emotional/cognitive, and academic. The medical domain assesses managing medications, booking appointments, disease literacy, self-management, and organ screening. The psychosocial domain assesses the adolescents' understanding of information related to adult rights, talking to providers as well as child motivation. Emotional concerns related to transition, self-advocacy skills, and completion of a personal health record. The academic domain assesses whether the adolescent is meeting school graduation requirements, able to verbalise an educational/job training plan and on track with future planning (eg, completed required testing). Items within each domain have equal value (ie, each question on the checklist is worth 1 point) and the sum of points yields the quantifiable assessment of how well patients are performing in each area of their health. Assessment meetings occur monthly when eligible patients are discussed. The questions are supported with illustrations explaining every answer. The tool was available in both paper and electronic formats. The children were also asked to rate the comprehensibility of the questionnaire on 0-10 numeric VAS. Sociodemographic, clinical as well as motivation scores were recorded.

Results: A total of 104 patients participated. The majority of patients (95.8\%) understood the questionnaire and completed it correctly, in self-administered modality. Mean comprehensibility score was $9.3 \pm 0.2$. Mean completion time was $6.2 \mathrm{~min}$, with little or no help $(78 \%)$. Children from low income families or those who had lower education level for their age needed more help. Internal consistency (Cronbach's alpha) for each domain score were $0.872,0.861,0.892$, and 0.884 respectively. Construct validity was demonstrated by testing different hypotheses $(p<0.01)$.

Conclusions: The developed illustrated questionnaire was a valid, patient-centred questionnaire which can be used by the paediatricians/rheumatologists to assess the level of preparedness of adolescents with JIA during planning for adult transition. The questionnaire gives information regarding the adolescents' ability to make appointments, attend their consultations, understand their treatment and to develop other skills required for transition to adult care. The questionnaire can be used to identify aspects in which the adolescent need education or training to achieve independence in transition-relevant skills. The questionnaire can be used also to set goals for the achievement of skills that will help adolescents manage their health and health care into adulthood. Implementing the questionnaire in the standard practice has the potential to improve transition assessment and support as well as improve health outcomes during healthcare transition for adolescents with inflammatory arthritic conditions.

Disclosure of Interest: None declared

DOI: 10.1136/annrheumdis-2018-eular.2795

\section{THU0603 \\ DISEASE MODIFYING ANTI-RHEUMATIC DRUGS IN JUVENILE IDIOPATHIC ARTHRITIS: MOVING TOWARDS TARGETED INDIVIDUALISED TREATMENT STRATEGY}

S.I. Nasef ${ }^{1}$, G. El Deriny ${ }^{2}$, M. Eissa ${ }^{3}$, M.A. Mortada ${ }^{4}$, W. Hassan ${ }^{5}$, D. Mekkawy ${ }^{6}$, Y. El Miedany ${ }^{6,7} .{ }^{1} R$ heumatology and Rehabilitation, School of Medicine, Suez Canal University, Ismaillia; ${ }^{2}$ Pediatrics, School of Medicine Alexandria University, Alexandria; ${ }^{3}$ Rheumatology and Rehabilitation, School of Medicine Cairo University, Cairo; ${ }^{4}$ Rheumatology and Rehabilitation, School of Medicine Zagazig university, Zagazig; ${ }^{5}$ Rheumatology and Rehabilitation, School of Medicine Banha University, Banha; ${ }^{6}$ Rheumatology and Rehabilitation, School of Medicine Ain Shams University, Cairo, Egypt, ${ }^{7}$ Rheumatology, Darent Valley Hospital, Dartford, UK

Background: Despite the expansion in the number of medications available in the treatment of juvenile idiopathic arthritis (JIA), inter-individual variation in therapeutic response and drug-associated toxicities continue to be a major concern and has driven efforts towards individualised therapy. Recent advances in paediatric treatment strategies and guidelines, as well as innovative approaches to identify a priori predictors of drug response, hold the promise for an individualised approach to therapy that will yield the highest efficacy and safety potential for each JIA patient.
Objectives: To determine whether baseline demographic, clinical, articular, laboratory variables and adherence to therapy may act as predictors of good response to DMARDs therapy in JIA.

Methods: Patients with JIA treated with DMARDs (methotrexate (MTX), leflunomide (LEF) or combination of MTX and LEF) were recruited for this study. Juvenile arthritis disease activity score (JADAS-27) was calculated at 3, 6 and 12 months Multivariate logistic regression analysis was used to identify predictors of good response according to JADAS-27 score, development of deformities and extraarticular manifestations (uveitis)

Results: A total of 114 children were included in this study. The majority of patients were females 91 (79.8\%). Mean age was $11.97 \pm 3.26$ years; mean age at disease onset was $8.19 \pm 3.46$ years whereas mean disease duration was 3.68 \pm 2.89 years. The most common ILAR subtype was polyarthritis RF negative (50.9\%), Polyarticular RF positive (21\%), oligoarticular extended (14.9\%), oligoarticular persistent $(7.9 \%)$ and systemic type (5.3\%). Over half of the patients were on MTX (55.3\%), and (34.2\%) were using combined MTX and LEF while (10.5\%) of patients were on LEF monotherapy. Mean MTX dose was $12.9 \pm 3.4 \mathrm{mg} / \mathrm{week}$ and mean LEF dose was $16.67 \pm 4.78 \mathrm{mg} /$ day. Prevalence of positive RF was 24/ $114(21.05 \%)$. Anti-nuclear antibody (ANA) was detected in 20/114 (17.5\%) of patients. $10.5 \%$ of patients had chronic anterior uveitis. At baseline, joint deformities were present in $(13.1 \%)$ of patients. After one-year follow up $(45.6 \%)$ of patients achieved remission while $(27.1 \%)$ reached a state of low disease activity. Multivariate logistic regression analysis revealed that the most important predictors of JADAS-27 remission/low disease activity status were: Short disease duration $<6.1 \pm 0.4$ months (OR 1.93), polyarticular disease subtype, Childhood Health Assessment Questionnaire (CHAQ) disability index <2.125 (OR 1.75), absence of joint damage/deformities (OR 1.92). A short duration of NSAIDs therapy $(<3$ months) before moving to MTX therapy as well as from MTX to combination of MTX and LEF therapy ( $<4$ months) were associated with the targeted disease activity status (OR 1.76 and 1.95 respectively). Adherence to therapy was also a predictor of good response (OR 1.86). 15.4\% of patients did not achieve the disease activity target and received biologic therapy.

Conclusions: The subgroup of JIA patients with polyarticular disease onset, shorter disease duration, rapid optimisation/escalation of DMARDs therapy as well as those who were adherent to therapy were significantly associated with a good response to DMARDs therapy.

Disclosure of Interest: None declared

DOI: 10.1136/annrheumdis-2018-eular.4156

\section{THU0604 JUVENILE INFLAMMATORY ARTHRITIS: THE DEVELOPMENT AND VALIDATION OF AN ILLUSTRATED QUESTIONNAIRE TO MEASURE CHILDREN'S MOTIVATION}

Y. El Miedany ${ }^{1,2}$, H. Lotfy ${ }^{3}$, N. El Aroussy ${ }^{2}$, D. Mekkawy ${ }^{2}$, S.I. Nasef ${ }^{4}$, W. Hassan ${ }^{5}$, M. Eissa ${ }^{6}$, G. El Deriny ${ }^{7}$, S. Almedany ${ }^{8}$, Y. Farag ${ }^{3}$, M. El Gaafary ${ }^{9}$, on behalf of PRINTO Egypt. ${ }^{1}$ Rheumatology, Darent Valley Hospital, Dartford, UK;

${ }^{2}$ Rheumatology and Rehabilitation, School of Medicine Ain Shams University; ${ }^{3}$ Pediatrics, School of Medicine Cairo University, Cairo; ${ }^{4}$ Rheumatology and Rehabilitation, School of Medicine Suez Canal University, Ismaillia; ${ }^{5}$ Rheumatology and Rehabilitation, School of Medicine Banha University, Banha; ${ }^{6}$ Rheumatology and Rehabilitation, School of Medicine Cairo University, Cairo; ${ }^{7}$ Pediatrics, School of Medicine Alexandria University, Alexandria; ${ }^{8}$ Rheumatology and Rehabilitation, School of Medicine Tanta University, Tanta; ${ }^{9}$ Community and Public Health, School of Medicine Ain Shams University, Cairo, Egypt

Background: Self-determination theory (SDT) is a macro theory of human motivation and personality that concerns people's inherent growth tendencies and innate psychological needs. SDT is concerned with the motivation behind choices people make without external influence and interference. JIA disrupts a child's sense of normality and impairs his or her motivation capacity. Children with JIA have a sense of being misunderstood and stigmatised, and they feel perpetually caught between having hope and control over their bodies and overwhelming pain and despair. To increase their confidence and the ability to manage pain, children need ongoing information about treatments, lifestyle management, and active involvement in their own health decision making.

Objectives: To develop a questionnaire for evaluating the "motivation" amongst children living with inflammatory arthritic conditions and assess the psychometric properties of that measure.

Methods: Based on content analysis and semi structured group discussion as well as Rasch analysis, ten domains were identified and used as a frame for an expert consensus and patient focus groups. The questionnaire was developed including: 10 -items scale ( $0-10$ on VAS scale). A mean score is calculated across all items. The total score ranged from $0-10$. Each item was supported by illustrations explaining both ends of the measure. The questionnaire was formatted, one copy for adolescents and another copy was directed for the parents of younger children. Construct validity was assessed by correlating the score of the questionnaire to disease activity scores (JADAS-27, SLEDAI-2K, ASDAS-CRP); 
measures of functional disability, quality of life, patient self-helplessness measure as well as the patients' adherence to therapy. Reliability and comprehensibility and sensitivity to change were also assessed.

Results: The questionnaire was assessed in 71 children with JIA, 32 with enthesitis related arthritis and 34 with cSLE. Results revealed that the developed illustrated questionnaire mean score correlated significantly with disease activity measures: JADAS-27: $r=-0.87$; ASDAS-CRP: $r=-0.85$, SLEDAI-2K: $r=-0.86$ reflecting its validity. It also correlated significantly with the scores of functional disability: $r=-0.89$, QoL: $r=-0.88$ as well as patient self-helplessness $r=-0.91$. The questionnaire was reliable (Cronbach's alpha 0.926 and had no misfitting items. The illustrations were well received and this was reflected on the questionnaire comprehensibility $(9.5)$ and sensitivity to change $(p<0.01)$. The patient motivation score showed significant $(p<0.01)$ correlation with adherence to therapy. Conclusions: The illustrated children motivation measure, is a patient-centred unidimensional scale that is valid, reliable and comprehensible. The measure has good psychometric properties indicating that it can be used at the individual child's level to tailor management and monitor changes in response to therapy. The illustrations enhanced the questionnaire perception by the children as well as the parents.

Disclosure of Interest: None declared

DOI: 10.1136/annrheumdis-2018-eular.2791

\section{THU0605 FACILITATING PATIENT CENTRED CARE: THE DEVELOPMENT OF ILLUSTRATED MULTIDIMENSIONAL PATIENT REPORTED OUTCOME MEASURE FOR CHILDREN WITH JUVENILE IDIOPATHIC ARTHRITIS}

Y. El Miedany $^{1,2}$, H. Lotfy ${ }^{3}$, N. El Aroussy ${ }^{2}$, D. Mekkawy ${ }^{2}$, S.I. Nasef ${ }^{4}$, W. Hassan ${ }^{5}$, G. El Deriny ${ }^{6}$, Y. Farag ${ }^{3}$, M. Eissa ${ }^{7}$, S. Almedany ${ }^{8}$, M. El Gaafary ${ }^{9}$, on behalf of PRINTO Egypt. ${ }^{1}$ Rheumatology, Darent Valley Hospital, Dartford, UK; ${ }^{2}$ Rheumatology and Rehabilitation, School of Medicine Ain Shams University: ${ }^{3}$ Pediatrics, School of Medicine Cairo University, Cairo; ${ }^{4}$ Rheumatology and Rehabilitation, School of Medicine Suez Canal University, Ismaillia; ${ }^{5}$ Rheumatology and Rehabilitation, School of Medicine Banha University, Banha; ${ }^{6}$ Pediatrics, School of Medicine Alexandria University, Alexandria; ${ }^{7}$ Rheumatology and Rehabilitation, School of Medicine Cairo University, Cairo; ${ }^{8}$ Rheumatology and Rehabilitation, School of Medicine Tanta University, Tanta; ${ }^{9}$ Community and Public Health, School of Medicine Ain Shams University, Cairo, Egypt

Background: The advances in paediatric rheumatology management have mandated a drastic change in the way children with juvenile arthritis are assessed and monitored. As a consequence, there has been a call for new outcome measures that reflect a more holistic approach to day to day standard management. Such an emphasis reflects contemporary views about the relation between mind and body, and acknowledges the critical link between physical and psychological health as well as adherence to therapy amongst the children living with inflammatory arthritis.

Objectives: To assess validity; reliability and responsiveness to change of an illustrated child/parent Multidimensional Patient Reported Outcome Measures questionnaire which can assess construct outcome measures of children with juvenile inflammatory arthritis.

Methods: 106 children with juvenile inflammatory arthritis were included in this work in a multicentre study. The questionnaire was developed by integrating information obtained from children living with JIA as well as their parents. The questionnaire included 5 main categories which are patient-centred: Health related quality of life: functional ability (children health assessment questionnaire) and quality of life (10-items reflecting psychological, social, school and behavioural issues as well as the patient's own perception). 2. Disease activity measure: pain intensity, the child's overall well-being, measure of fatigue and morning stiffness using (0-10 numerical visual analogue scale), 3. self-reported joint tenderness and swelling. 4. Current medication, side effects as well as adherence to therapy (2 questions using 0-10 numerical visual analogue scale); 4. Comorbidities as well as 5 . Patient motivation. All the items were supported by illustrations to explain the question and make it easier to understand. The questionnaire has parent and patient versions. The disease activity status was assessed using JADAS-27.

Results: The questionnaire was reliable as demonstrated by a high-standardised alpha $(0.890-0.978)$. The questionnaire items correlated significantly $(p<0.01)$ with clinical parameters of disease activity. The patient reported tender joints correlated significantly with the physician's scores $(0.842)$. Changes in functional disability, quality of life as well as the motivation score showed significant variation $(p<0.01)$ with diseases activity status in response to therapy. The illustrated PROMs questionnaire showed also a high degree of comprehensibility (9.6).

Conclusions: Integrating patient reported outcome measures into standard clinical practice is feasible and applicable. This version of illustrated multidimensional questionnaire was found to be valid and reliable. It provides informative quantitative measure for the disease activity core set data, and in the meantime, facilitates assessing the children's health related quality of life measure, adherence to therapy, comorbidities as well as motivation on individual basis.

Disclosure of Interest: None declared

DOI: 10.1136/annrheumdis-2018-eular.2789

\section{THURSDAY, 14 JUNE 2018 \\ Other orphan diseases}

\section{THU0606 \\ EFFECT OF INTERLEUKIN-1 ANTAGONISTS ON THE QUALITY OF LIFE IN FAMILIAL MEDITERRANEAN FEVER PATIENTS}

Ö. Varan ${ }^{1}$, H. Küçük ${ }^{2}$, H. Babaoğlu' ${ }^{1}$, N. Atas ${ }^{1}$, H. Satıs ${ }^{1}$, R. Bilici Salman ${ }^{1}$, A. Tufan ${ }^{1}$, B. Göker ${ }^{1}$, S. Haznedaroğlu' ${ }^{1}$, M.A. Öztürk ${ }^{1} .{ }^{1}$ Rheumatology, Gazi University, Ankara; ${ }^{2}$ Rheumatology, Education and Training Hospital, erzurum, Turkey

Background: Familial Mediterranean Fever (FMF) is characterised by febrile inflammatory painful attacks of peritonitis, pleuritis, arthritis and erzypeloid erythema. Colchicine is the mainstay of treatment in FMF but about\%10 patients do not respond well to colchicine. IL-1 antagonists have been shown to be effective in the prevention of attacks in colchicine resistant FMF (crFMF) patients. Herein we investigated effect of these agents on quality of life of crFMF patients.

Objectives: to investigate effect of IL-1 antagonists on quality of life of crFMF patients.

Methods: Data is derived from Gazi FMF cohort which was established in year 2010. Since then data of patients with FMF who were diagnosed according to the Tel Hashomer criteria were registered. Co-morbidities, detailed attack characteristics, treatments, laboratory parameters and impact of FMF on their life in terms of quality of life were recorded. A retrospective cohort analysis was made from records of patients who were treated with IL-1 inhibitors. SF-36 form was filled before and 3 months after the IL-1 antagonist treatment. Wilcoxon test was used for the analyses and a p value equal or less than 0.05 is considered as statistically significant.

Results: there were 41 patients ( 24 women and 17 men). Anakinra was used in 33 patients and 8 patients received canakimumab. There was a statistically significant reduction in the frequency, severity and the duration of attacks after treatment with IL-1 antagonists ( $\mathrm{p}<0.001$, for each). A statistically significant improvement was observed in all domains of SF-36 (figure 1).

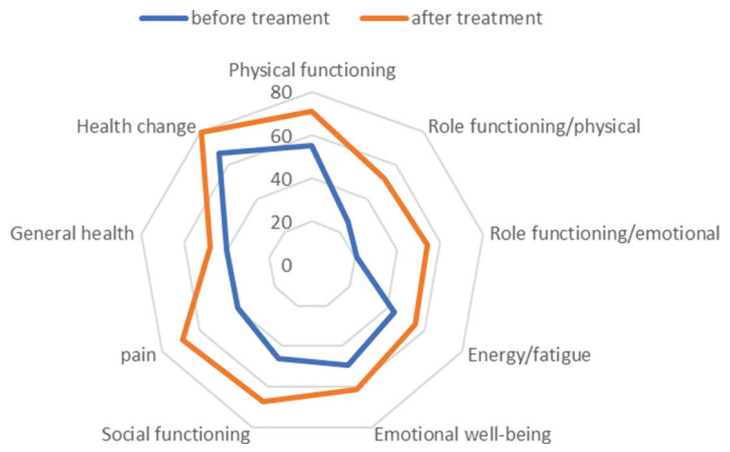

Figure. The change of SF-36 parameters before and after treatment

Conclusions: IL-1 inhibitor therapy reduces frequency, severity and duration of attacks and significantly improves the quality of life of crFMF patients.

Disclosure of Interest: None declared

DOI: 10.1136/annrheumdis-2018-eular.6495

\section{THU0607 AUTOINFLAMMATORY SYNDROMES: CLINICAL AND GENETIC CHARACTERISATION OF A COHORT OF ADULT PATIENTS IN A SINGLE REFERENCE CENTRE}

A. Ruiz Roman ${ }^{1}$, S. Garcia Morillo², M.A. Montes Cano ${ }^{3}$, M. Lisbona Muñoz ${ }^{1}$ C. Aguilera Cros ${ }^{1}$, A. Muñoz Jimenez ${ }^{1}$, E. Rubio Romero ${ }^{1} .{ }^{1}$ Reumatologia; ${ }^{2}$ Medicina Interna; ${ }^{3}$ Inmunologia, Hospital Universitario Virgen del Rocio, Sevilla, Spain

Background: Autoinflammatory syndromes are diseases that are characterised by increased inflammation mediated by cells and molecules of the innate immune system. Currently, new techniques have been developed that allow earlier diagno sis and treatment of these pathologies. 